ARTICLE

https://doi.org/10.1038/s41467-020-14481-z

\title{
Atomic structure and defect dynamics of monolayer lead iodide nanodisks with epitaxial alignment on graphene
}

\author{
Sapna Sinha (1) ${ }^{1}$, Taishan Zhu ${ }^{2}$, Arthur France-Lanord (1) ${ }^{2}$, Yuewen Sheng (1) ${ }^{1}$, Jeffrey C. Grossman (1) ${ }^{2}$, \\ Kyriakos Porfyrakis (i) $^{3}$ \& Jamie H. Warner ${ }^{4 \star}$
}

Lead lodide $\left(\mathrm{Pbl}_{2}\right)$ is a large bandgap 2D layered material that has potential for semiconductor applications. However, atomic level study of $\mathrm{Pbl}_{2}$ monolayer has been limited due to challenges in obtaining thin crystals. Here, we use liquid exfoliation to produce monolayer $\mathrm{Pbl}_{2}$ nanodisks (30-40 nm in diameter and $>99 \%$ monolayer purity) and deposit them onto suspended graphene supports to enable atomic structure study of $\mathrm{Pbl}_{2}$. Strong epitaxial alignment of $\mathrm{Pbl}_{2}$ monolayers with the underlying graphene lattice occurs, leading to a phase shift from the $1 \mathrm{~T}$ to $1 \mathrm{H}$ structure to increase the level of commensuration in the two lattice spacings. The fundamental point vacancy and nanopore structures in $\mathrm{Pbl}_{2}$ monolayers are directly imaged, showing rapid vacancy migration and self-healing. These results provide a detailed insight into the atomic structure of monolayer $\mathrm{Pbl}_{2}$, and the impact of the strong van der Waals interaction with graphene, which has importance for future applications in optoelectronics.

\footnotetext{
${ }^{1}$ Department of Materials, University of Oxford, 16 Parks Road, Oxford OX1 3PH, UK. ${ }^{2}$ Department of Materials Science and Engineering, Massachusetts Institute of Technology, 77 Massachusetts Avenue, Cambridge, MA 02139, USA. ${ }^{3}$ Faculty of Engineering and Science, University of Greenwich, Central Avenue, Chatham Maritime, Kent, ME4 4TB, UK. ${ }^{4}$ Department of Mechanical Engineering, University of Texas at Austin, 204 Dean Keeton Street, Austin 78712, USA. *email: jamie.warner@austin.utexas.edu
} 
T wo-dimensional (2D) materials attract interest because of their unique chemical and physical properties, facilitating the study of novel physics, e.g., trions, valley polarization, etc $^{1-3}$. Although graphene exhibits an exceptionally high carrier mobility $\left(>10^{6} \mathrm{~cm}^{2} \mathrm{~V}^{-1} \mathrm{~s}^{-1}\right.$ at $2 \mathrm{~K}$ ), its zero bandgap poses difficulties for many semiconductor applications ${ }^{4}$. Monolayer transition metal dichalcogenides (TMDs), such as $\mathrm{MoS}_{2}$, can have direct band gaps of $\sim 1.8 \mathrm{eV}$, but do not exhibit very high charge carrier mobility $^{5-7}$. For Mo- and W-based TMDs, such as $\mathrm{MoSe}_{2}$, $\mathrm{WS}_{2}, \mathrm{WSe}_{2}$, etc., the band gaps fall within the range of $1.0-2.0 \mathrm{eV}$, which is the red to near infrared regions ${ }^{8,9}$. For optoelectronics, blue, green, and ultraviolet light-emitting diodes are also needed for full color displays and cameras. Currently, there are not many experimental studies of 2D materials that can satisfy the demands for the green to UV spectral regions and more research is needed to expand this area.

$\mathrm{PbI}_{2}$ is a layered direct bandgap semiconductor with bandgap of $2.4 \mathrm{eV}$ in its bulk form, whereas its $2 \mathrm{D}$ monolayer has an indirect bandgap of $\sim 2.5 \mathrm{eV}$, with possibilities to tune the bandgap between $1-3 \mathrm{eV}^{10-13} \cdot \mathrm{PbI}_{2}$ is frequently used to fabricate organic-inorganic halide perovskite solar cells ${ }^{14,15}$, and as a highenergy photon detector material for gamma-rays and X-rays ${ }^{16-18}$. $\mathrm{PbI}_{2}$ has a wide variety of I-Pb-I stacking and this gives rise to more than a dozen polymorphs ${ }^{19}$. However, the thickness of each single layer $(0.7 \mathrm{~nm})$, and the distance between each lead and iodide atoms $(0.32 \mathrm{~nm})$ is independent of the polytypes ${ }^{19}$. The $2 \mathrm{H}$ structure is the most commonly found polytype of threedimensional $\mathrm{PbI}_{2}$, where each plane of $\mathrm{Pb}$ and I atoms are shifted with respect to each other and form overlapping hexagons.

Layered $\mathrm{PbI}_{2}$ has been shown to be excellent candidate for use in optoelectronic applications, photodetectors, and photon detection ${ }^{20-23}$. Ultrathin $\mathrm{PbI}_{2}$ is an interesting system for studying quantum-confinement effects because the exciton-phonon couplings are dependent on the degree of localization of electronic charge ${ }^{24,25}$. Cabana et al. ${ }^{26}$ fabricated $\mathrm{PbI}_{2}$ interfaces with carbon nanotubes and studied the change in the density of states of the system. Zhou et al. ${ }^{10}$ studied the graphene/ $\mathrm{PbI}_{2}$ van der Waals interface and predicted $1.5 \mathrm{eV}$ increase in the visible light absorption capability of the heterostructure as compared to pure $2 \mathrm{D} \mathrm{PbI}_{2}$. Recent work on few layer shows $\mathrm{PbI}_{2}$ as a promising candidate for application in the field of ultrafast saturable absorbers $^{27}$. However, research into the atomic structure of monolayer $\mathrm{PbI}_{2}$ has been limited to date because of the challenges in obtaining high-quality monolayer crystals and preparing suitable samples for transmission electron microscopy studies. Further work is needed to reveal the structure and dynamics of edges, point defects, vacancy clusters, and nanopores in $\mathrm{PbI}_{2}$ monolayer and its interaction with other $2 \mathrm{D}$ crystals, such as graphene.

Here, we used liquid phase exfoliation (LPE) to isolate $\mathrm{PbI}_{2}$ monolayer flakes from a starting bulk $\mathrm{PbI}_{2}$ powder. Liquid-phase exfoliation is one of the simplest methodologies for producing $2 \mathrm{D}$ materials on a large scale $28-30$. In the past decades, various research has been carried out to find suitable solvents based on their interactions with the 2D material to produce suspended 2D monolayer crystals. Surface tension, the Hilderbrand solubility parameter, the Hansen solubility parameter, surface tension components, etc. have been the widely used parameters to screen appropriate solvents ${ }^{31-35}$. Using these parameters, we screened the commonly used solvents and found chloroform $\left(\mathrm{CHCl}_{3}\right)$ to produce monolayer $2 \mathrm{D} \mathrm{PbI}_{2}$ crystals. We used annular dark-field scanning transmission electron microscopy (ADF-STEM) to studying the $\mathrm{PbI}_{2}$ atomic structure, by depositing it from solution onto a suspended graphene support and allowing it to dry ${ }^{36-38}$. The high electron transparency of graphene, enables provides excellent contrast from the $\mathrm{Pb}$ and I atoms in ADF-STEM. We report on the fundamental atomic structure, point vacancies, vacancy clusters, vacancy dynamics, edge terminations and edge etching, and the epitaxial interactions with the underlying graphene support.

\section{Results}

Synthesis. $\mathrm{PbI}_{2}$ is a unique exception to all the metal halide compounds that show $\mathrm{CdI}_{2}$ structure, in that it has the largest metal halide bond length ${ }^{39}$. As a result, the bonds are not as ionic as that of the other compounds that also show $\mathrm{CdI}_{2}$ crystal structure, such as $\mathrm{MgI}_{2}, \mathrm{FeBr}_{2}$, etc. However, it is sufficiently ionic to dissolve into polar solvents, such as dimethylformamide (DMF), n-methyl-2-pyrrolidone (NMP), dimethyl sulfoxide (DMSO), and to some extent, water ${ }^{40,41}$. Recent research on Lewis basicity of solvents, also quantified by Gutmann's donor number $-D_{n}$, has shown that solvents that solubilize the precursor $\left(\mathrm{PbI}_{2}\right)$ at a total concentration of $1 \mathrm{M}$, have higher $D_{n}$ values of $>25$ (ref. ${ }^{42}$ ). Previous results on the liquid-phase exfoliation for other $2 \mathrm{D}$ materials have shown that the most efficient solvents have $\mathrm{H}$-bonding components so as to maximize the dispersability of the material in the solution, which is in agreement with the Hansen solubility parameter theory 43,44 . We explored several different solvents and found that chloroform serves as an excellent medium to exfoliate $\mathrm{PbI}_{2}$. $\mathrm{CHCl}_{3}$ has low dipole moment $(\mu=1.04)$, low dielectric constant $\left(\epsilon_{T}=20^{\circ} \mathrm{C}=\right.$ 4.8 ), low donor number $\left(D_{n}=4\right)$, as well as has the ability to form hydrogen bonds ${ }^{45-47}$. In addition, $\mathrm{CHCl}_{3}$ has a very low boiling point of $61^{\circ} \mathrm{C}$ which facilitates in quick and easy removal after solution dropcasting on to substrates.

Figure 1a shows the schematic diagram of the liquid exfoliation experimental procedure. The sonication time is important for controlling the exfoliation of $\mathrm{PbI}_{2}$. Less sonication leaves multilayer $\mathrm{PbI}_{2}$ dispersed in the solution, whereas longer sonication time renders very small flakes or completely destroys the sheets (Supplementary Note 1). Moreover, the supernatant used for dropcasting also plays a role, and only the solution from the top has been used (Supplementary Note 2). Our results show successful exfoliation of mostly monolayer flakes of the $\mathrm{PbI}_{2}$ from its bulk counterpart. Moreover, the dispersed solution was found to be stable for a considerable number of days. Even after a week of the sonication process, we could still observe monolayer flakes dispersed in the solution (Supplementary Note 1). Out of $>300$ flakes studied in this experiment, we only found 2 flakes with bilayer structure (Supplementary Note 3), whereas all others were monolayers. Some nanoparticles that are observed come from the graphene underneath and not from $\mathrm{PbI}_{2}$ itself (Supplementary Note 4). Figure $1 \mathrm{~b}-\mathrm{d}$ shows the ADF-STEM images of the monolayer $\mathrm{PbI}_{2}$ suspended on monolayer graphene on a TEM grid at various magnifications. The length and width of $\sim 300$ flakes was measured (Fig. 1e, f), giving $\sim 42$ and $28 \mathrm{~nm}$, respectively, with bigger flakes ranging to $>160 \mathrm{~nm}$ in size (Fig. 1g).

1-H structural phase. The bulk crystal structure of $\mathrm{PbI}_{2}$ has a $\mathrm{CdI}_{2}$ structural type with lead and iodide layers stacked together in $\mathrm{ABC}$ instead of ABA stacking. However, in the monolayer form there are two structural phases, namely-1-H phase and 1-T phase. $\mathrm{PbI}_{2}$ has similar structures to the TMDs $\left(\mathrm{MoS}_{2}\right.$ and $\left.\mathrm{WS}_{2}\right)$, where the metal $(\mathrm{Pb})$ atomic plane is in the middle and the two iodide atomic layers at the top and the bottom. A monolayer from a $2 \mathrm{H} \mathrm{PbI}_{2}$ bulk crystal structure (Fig. 2a) of $\mathrm{PbI}_{2}$ typically has a 1-T phase (Fig. 2b), where the two iodide atoms do not share the same $z$-axis and are located in an octahedral coordination. Whereas the $1-\mathrm{H}$ phase of monolayer $\mathrm{PbI}_{2}$ (Fig. 2e, $\mathrm{f}$ ) has two iodide atomic layers on top of each other in a trigonal-prismatic 

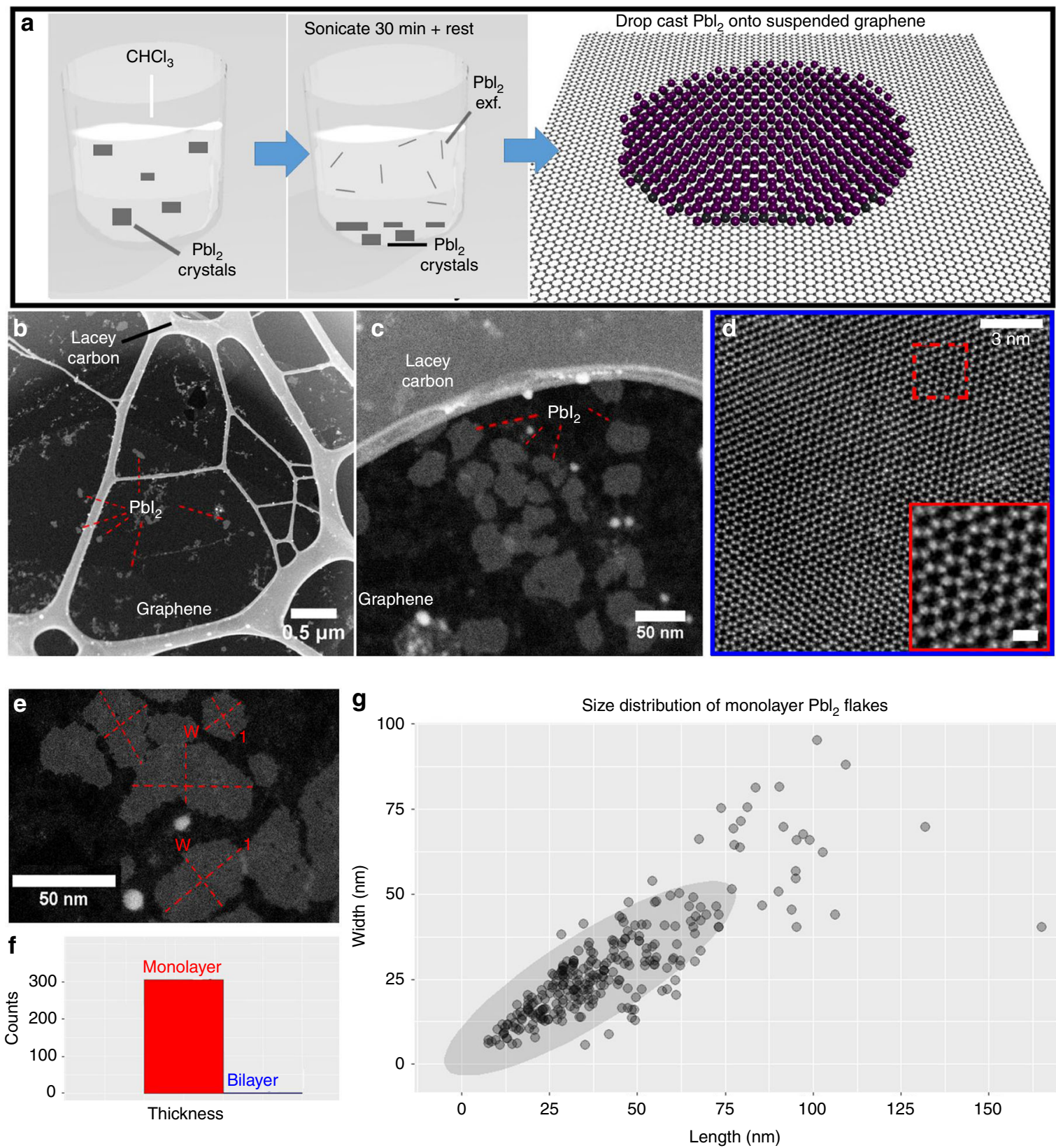

Fig. 1 Schematic diagram for liquid exfoliation and results. a Schematic of the LPE synthesis of monolayer $\mathrm{Pbl}_{2}$ flakes and deposition on to suspended graphene. b, c ADF-STEM image of monolayer $\mathrm{Pbl}_{2}$ flakes at different magnifications. d Atomic resolution ADF-STEM image of a monolayer Pbl 2 flake. The inset in the figure shows an atomic resolution image of the structure. The scale bar is $0.5 \mathrm{~nm}$. e Typical method for measuring the length(I) and width ( $\mathrm{W}$ ) of the monolayer flake. $\mathbf{f}$ Histogram showing the thickness distribution of $\mathrm{Pbl}_{2}$ flakes. $\mathbf{g}$ Plot of the size distribution for $>300$ monolayer flakes. Overlapping sizes of different flakes show darker color for their marker dots. The oval represents the highest congregation of flake sizes in the overall sample distribution.

coordination, as shown in Fig. 2e, f respectively. The position of the $\mathrm{Pb}$ and $\mathrm{I}$ atoms can be determined by their contrast in ADFSTEM images. $\mathrm{Pb}$ has $Z=82$, I has $Z=53$, and $2 \mathrm{I}$ has $Z=106$, which means that the 2 I positions will have higher contrast than the single $\mathrm{Pb}$. The line profiles (Fig. $2 \mathrm{~d}, \mathrm{~h}$ ) of the multislice image simulations for $1 \mathrm{~T}$ and $1 \mathrm{H} \mathrm{PbI}_{2}$ monolayers give the distance across $1->2$ and $3->4$ in Fig. $2 c$, g, respectively, for comparison to our experimental data (Fig. 2i-k). The experimental $\mathrm{Pb}-\mathrm{Pb}$ distance of $1.027 \mathrm{~nm}$ and the inter-atomic $\mathrm{Pb}-2 \mathrm{I}$ distances are in excellent agreement with the simulated distances of the $1 \mathrm{H}$ structural type (Fig. 2h). To further validate the case of monolayer $1 \mathrm{H}$ structure, we bombarded the nanodisks with electron beam.
And as seen in Supplementary Note 5, post few minutes of beam exposure, the agglomeration of vacancies lead to hole formation. Previous studies have reported that the initial defect or hole formation occurs layer-wise instead ${ }^{38,48}$. Since, the hole formation revealed no further layers underneath, it is conclusive to say that this is a monolayer structure instead of $\mathrm{AA}^{\prime}$-stacked bilayer $1 \mathrm{H}$ structure.

The $1 \mathrm{~T}$ phase is expected to be more stable than the $\mathrm{H}$ phase with total energy $165 \mathrm{meV}$ per unit cell lower ${ }^{49}$, unlike the other $2 \mathrm{D}$ materials such as $\mathrm{MoS}_{2}$, where the $1 \mathrm{H}$ phase is generally known to be more stable than the $1 \mathrm{~T}$ phase ${ }^{50}$. However, all $\mathrm{PbI}_{2}$ flakes suspended on graphene, from smallest $3 \mathrm{~nm}$ to larger 

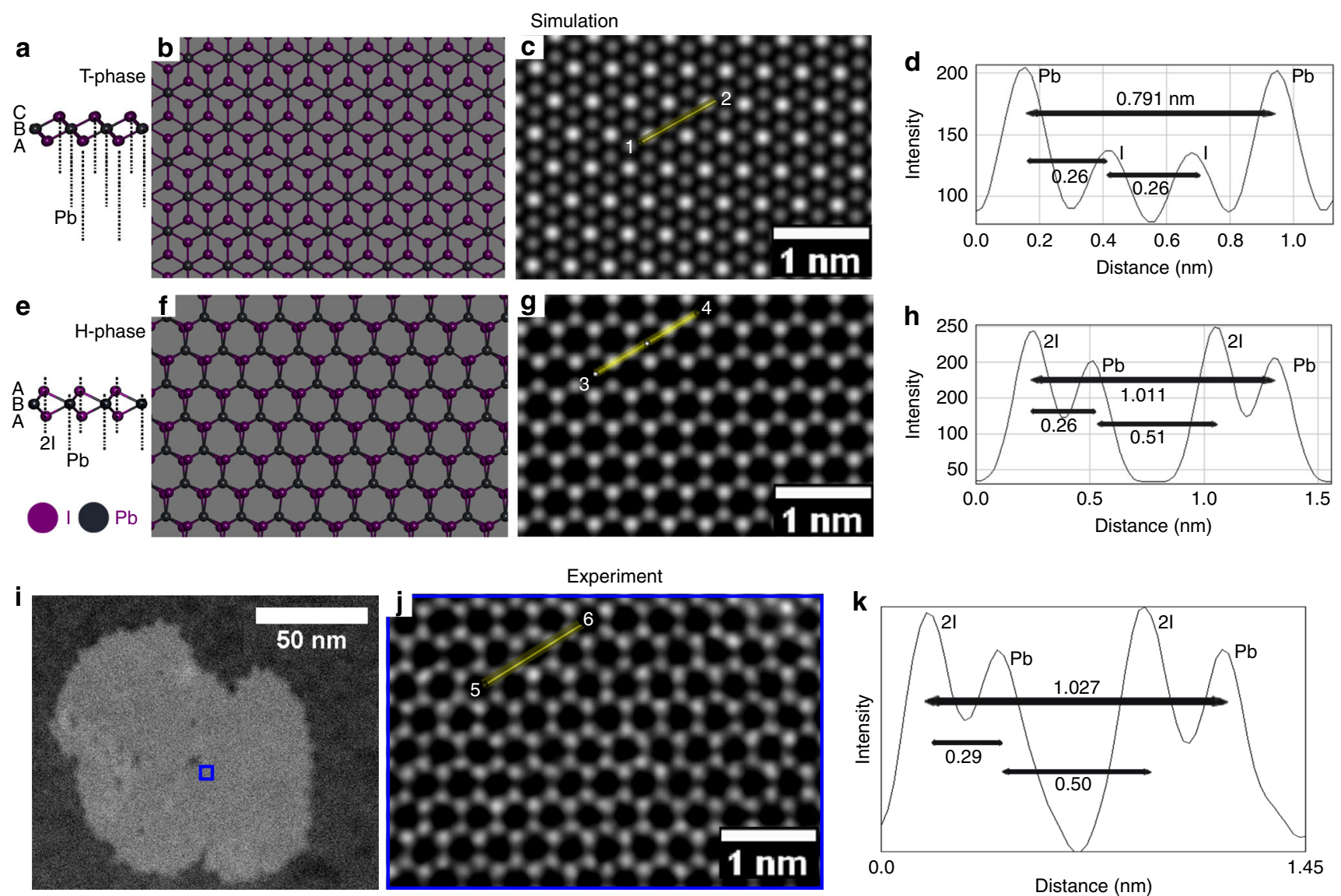

Fig. 2 Atomic resolution ADF-STEM analysis of monolayer $\mathbf{1 ~} \mathbf{H}$ and $1 \mathbf{T}$ phase. a Side view of the $1 \mathrm{~T}$ atomic structure of $\mathrm{Pbl}_{2}$. $\mathbf{b}$ Top view of the $1 \mathrm{~T}$ atomic model of single layer $\mathrm{Pbl}_{2}$. c Multislice ADF-STEM image simulation of the $1 \mathrm{~T}$ atomic model in $\mathbf{b}$. $\mathbf{d}$ Line profile of the lead and iodide intensities marked with yellow line in the atomic model in $\mathbf{c}$, along the direction 1-2. e Side view of $1 \mathrm{H}$ monolayer atomic crystal structure of $\mathrm{Pbl}_{2}$. $\mathbf{f} \mathrm{Top}$ view of the $1 \mathrm{H}$ atomic model of single layer $\mathrm{Pbl}_{2} . \mathbf{g}$ Multislice ADF-STEM image simulation of the $1 \mathrm{H}$ atomic model shown in $\mathbf{f}$. $\mathbf{h}$ Line profile of the lead and iodide intensities marked with yellow line in the atomic model in $\mathbf{g}$, along the direction 3-4. $\mathbf{i}$ Low-magnification ADF-STEM image of a monolayer Pbl ${ }_{2}$ flake suspended on graphene. $\mathbf{j}$ Higher magnification of the region indicated by the blue box in $\mathbf{i}$, showing the $1 \mathrm{H}$ atomic structure. $\mathbf{k}$ Line profile taken from region marked by the yellow box in $\mathbf{j}$, which corresponds to same position with the region marked in simulation $\mathbf{g}$, along the direction 5-6.

hundreds of nanometers in size (Supplementary Note 6), showed $1 \mathrm{H}$ phase, attributed to epitaxial interactions with the underlying graphene support. Chemical vapor deposition (CVD)-grown graphene has grain boundaries with random orientation and hence, we saw that $\mathrm{PbI}_{2}$ flakes within a region $(1-5 \mu \mathrm{m})$ showed the same relative crystal orientation relative to the graphene crystal (Supplementary Note 7, Supplementary Note 8$)^{50,51}$. To confirm the case of $\mathrm{PbI}_{2}-$ graphene interactions, we deposited $\mathrm{PbI}_{2}$ flakes directly on top of lacey carbon TEM grids without graphene, and observed $\mathrm{PbI}_{2}$ flakes with $1 \mathrm{~T}$ structure instead (Supplementary Note 9). We also carried a time-dependent study and concluded that the spatial variations in the $\mathrm{PbI}_{2}$ lattice occurred during the early stages after deposition on graphene, eventually completely transforming to well-defined $1 \mathrm{H}$ phase over time (Supplementary Note 10). After 1 day, all the $\mathrm{PbI}_{2}$ flakes on graphene showed well-defined $1 \mathrm{H}$ phase with the same orientations in a given area. To understand why $\mathrm{PbI}_{2}$ adopts $1 \mathrm{H}$ structure with concomitant alignment to the armchair direction of graphene, we examine the Moire patterns formed between the two crystals (Supplementary Note 11). The best lattice match occurs when the $\mathrm{PbI}_{2}$ adopts $1 \mathrm{H}$ phase and is aligned to the armchair direction, which agrees with our experimental findings.

Interestingly, we also found that the crystal orientation of $\mathrm{PbI}_{2}$ on graphene had preferred orientation of $\mathrm{PbI}_{2}$ zigzag aligned to graphene armchair direction, with only a small fraction showing the zigzag to zigzag alignments. Figure $3 \mathrm{a}$, $\mathrm{f}$ shows the ADFSTEM images and their FFTs of the two different types of orientations of $\mathrm{PbI}_{2}$ on graphene. Figure 3k-r shows the atomic models of $\mathrm{PbI}_{2}$ on graphene for the two aligned directions and the multislice image simulation, along with the fast Fourier transform (FFT) results matching the experimental data. To understand why $\mathrm{PbI}_{2}$ adopts $1 \mathrm{H}$ structure with concomitant alignment in these particular directions of graphene, we employed density functional theory (DFT) calculations to study the structural and energetic stability of the different alignments. We used a shortrange relaxed atomic model of $\mathrm{PbI}_{2}$ on graphene, as shown in Fig. 3s, and rotated it every 5 degrees (Fig. 3s) to find the most energetically stable heterostructure (Fig. 3t). This also confirms our results to how the graphene influences the stability of the $\mathrm{PbI}_{2}$ and leads to these energetically favorable structure that are also observed in the experimental results.

Structural defects. Next, we examine the edge terminations of $\mathrm{PbI}_{2}$, because edges of $2 \mathrm{D}$ materials can influence the electrical, chemical, and catalytic properties due to the different bonding (angles, bond distances, etc.) between atoms $\mathrm{s}^{51-54}$. Fig. $4 \mathrm{a}$ shows the typical edges of two different monolayer $\mathrm{PbI}_{2}$ flakes, just after being deposited on the graphene. The edges are rough and do not 

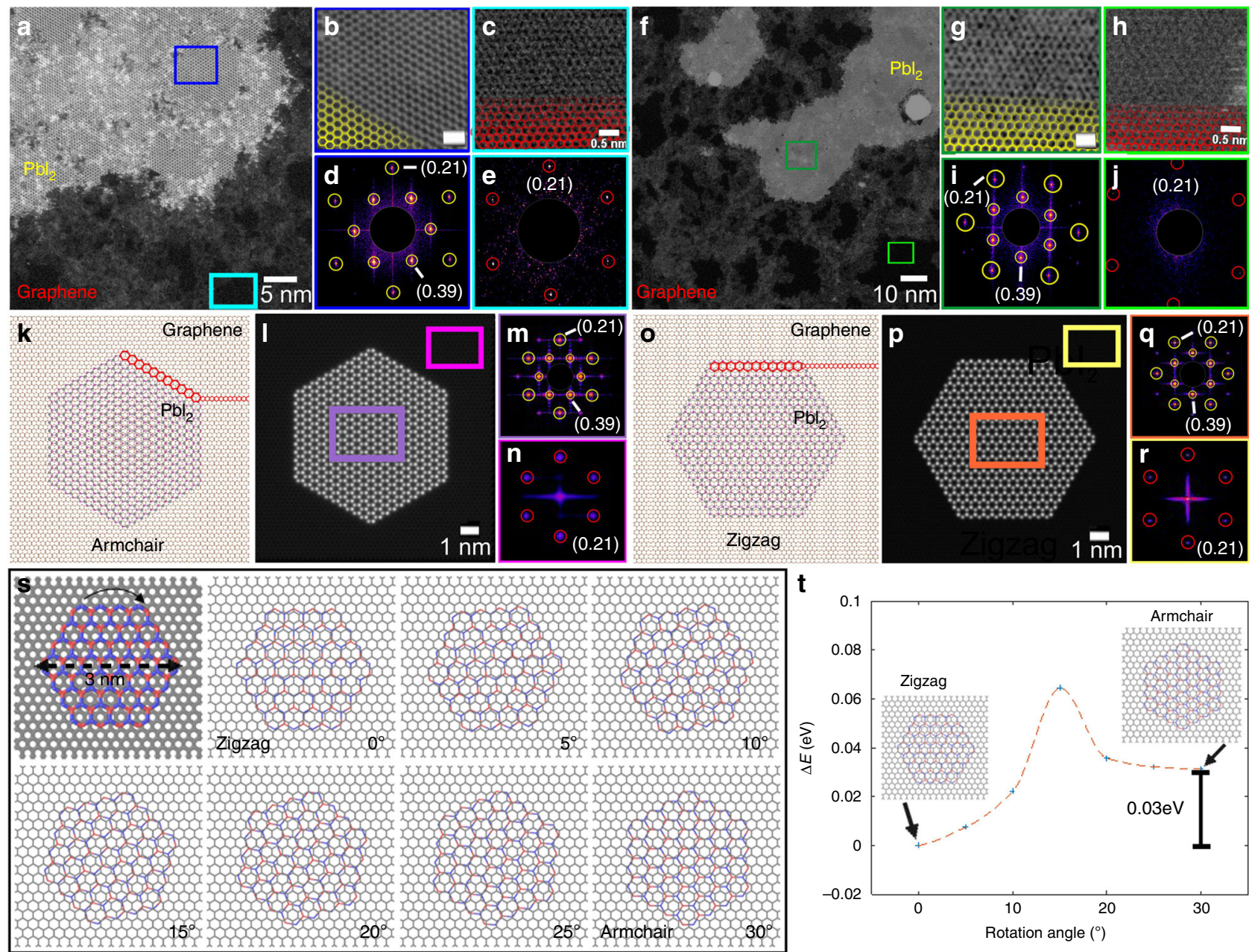

Fig. 3 Crystal orientation of monolayer $\mathbf{P b l}_{\mathbf{2}}$ on graphene. a ADF-STEM images of $\mathrm{Pbl}_{2}$ flake suspended on graphene. $\mathbf{b}$ High-resolution image of $\mathrm{Pbl}{ }_{2}$ crystal annotated in the dark blue box in $\mathbf{a}$. Scale bar corresponds to $1 \mathrm{~nm}$. $\mathbf{c}$ High-resolution image of graphene taken from the cyan box region indicated in a. $\mathbf{d}$ FFT of $\mathrm{Pbl}_{2}$ from the region indicated in $\mathbf{b}$. $\mathbf{c}$ FFT of graphene from the region indicated in $\mathbf{c}$. $\mathbf{f}$ ADF-STEM images of $\mathrm{Pbl}_{2}$ flake suspended on graphene. $\mathbf{g}$ High-resolution image of $\mathrm{Pbl}_{2}$ crystal indicated by the dark green box in $\mathbf{f}$. Scale bar corresponds to $1 \mathrm{~nm}$. $\mathbf{h}$ High-resolution image of graphene from the light green box in $\mathbf{f}$. i FFT of $\mathrm{Pbl}_{2}$ from the region indicated in $\mathbf{g}$. $\mathbf{h}$ FFT of graphene from the region indicated in $\mathbf{f}$. $\mathbf{k}$ Atomic model of $\mathrm{Pbl} 2(1 \mathrm{H})$ on graphene in the armchair direction. I Multislice image simulations based on the atomic model in $\mathbf{k} . \mathbf{m}$ FFT of $\mathrm{Pbl}_{2}$ from the region indicated by the purple box in $\mathbf{I}$. $\mathbf{n}$ FFT of graphene from the region indicated by the pink box in $\mathbf{I}$. o Atomic model of $\mathrm{Pbl}_{2}(1 \mathrm{H})$ on graphene in the armchair direction. $\mathbf{p}$ Multislice image simulations based on the atomic model in $\mathbf{k}$. $\mathbf{q}$ FFT of $\mathrm{Pbl}_{2}$ from the region indicated by the orange box in $\mathbf{p}$. $\mathbf{r}$ FFT of graphene from the region indicated by the yellow box in $\mathbf{p}$. $\mathbf{s}$ Atomic models showing $\mathrm{Pbl}_{2}$ relative to graphene for $\mathbf{s}$ DFT-relaxed atomic model of $\mathrm{Pbl}_{2}$ on graphene, rotated every 5 degrees. $\mathbf{t}$ Energy profile of various orientation angles of the $\mathrm{Pbl}_{2}$ flake on graphene.

appear to be highly faceted to a specific crystal lattice direction. After electron beam irradiation (Fig. 4b), the edges are etched to form sharp zigzag-faceted terminations (Fig. 4c). The zigzag edges have iodide atoms protruding out on one side and lead atoms on the adjacent edge of the same flake (Fig. 4d, e). Metal and chalcogenide-terminated zigzag edges have been routinely observed for as grown monolayer TMDs, such as $\mathrm{MoS}_{2}{ }^{55,56}$. However, in this case, the edge etching also happened in directions which maintained the zigzag- $\mathrm{PbI}_{2}$ alignment to the armchair graphene direction (Supplementary Note 12).

Edge etching occurred by atomic loss under the electron beam (Fig. 4h-i), and edges also showed atomic attachment to restructure (Fig. 4j-l, Supplementary Note 13). After $30 \mathrm{~s}$ of electron beam exposure, the atoms at the edge are sputtered out, by unzipping the outer most atoms to retain its uniform zigzag termination (Fig. $4 \mathrm{i})^{56}$. Prolonged exposure to the electron beam eventually leads to edge roughening. DFT and ab initio molecular dynamics simulations have shown the edge reconstructions effect the electrical and magnetic properties of the material ${ }^{57}$.

ADF-STEM of the central lattice region of the $\mathrm{PbI}_{2}$ monolayers revealed point defects across the region (Fig. 5a). Single-point vacancies were observed for both the lead and iodide sub-lattice sites in $\mathrm{PbI}_{2}$ (Fig. 5b, c), and identified using line profiles (Supplementary Note 14). The energy to remove a single iodide or lead atom from the lattice into the vacuum was calculated by DFT to be 3.15 and $6.36 \mathrm{eV}$, respectively (Supplementary Note 15). The maximum amount of energy $(E)$ transferred by an incident electron (@80 keV) to a single iodide or lead atom is calculated to be 1.38 and $0.85 \mathrm{eV}$, respectively ${ }^{58}$. This energy transfer from the incident electron to the specimen atoms is much lower than the required sputtering energy calculated by DFT. Hence, we can deduce that the mechanism of damage in this system is more complicated than purely knock-on damage from atomic sputtering, and likely involves radiolysis. This is not surprising, since $\mathrm{PbI}_{2}$ is a large bandgap semiconductor and 

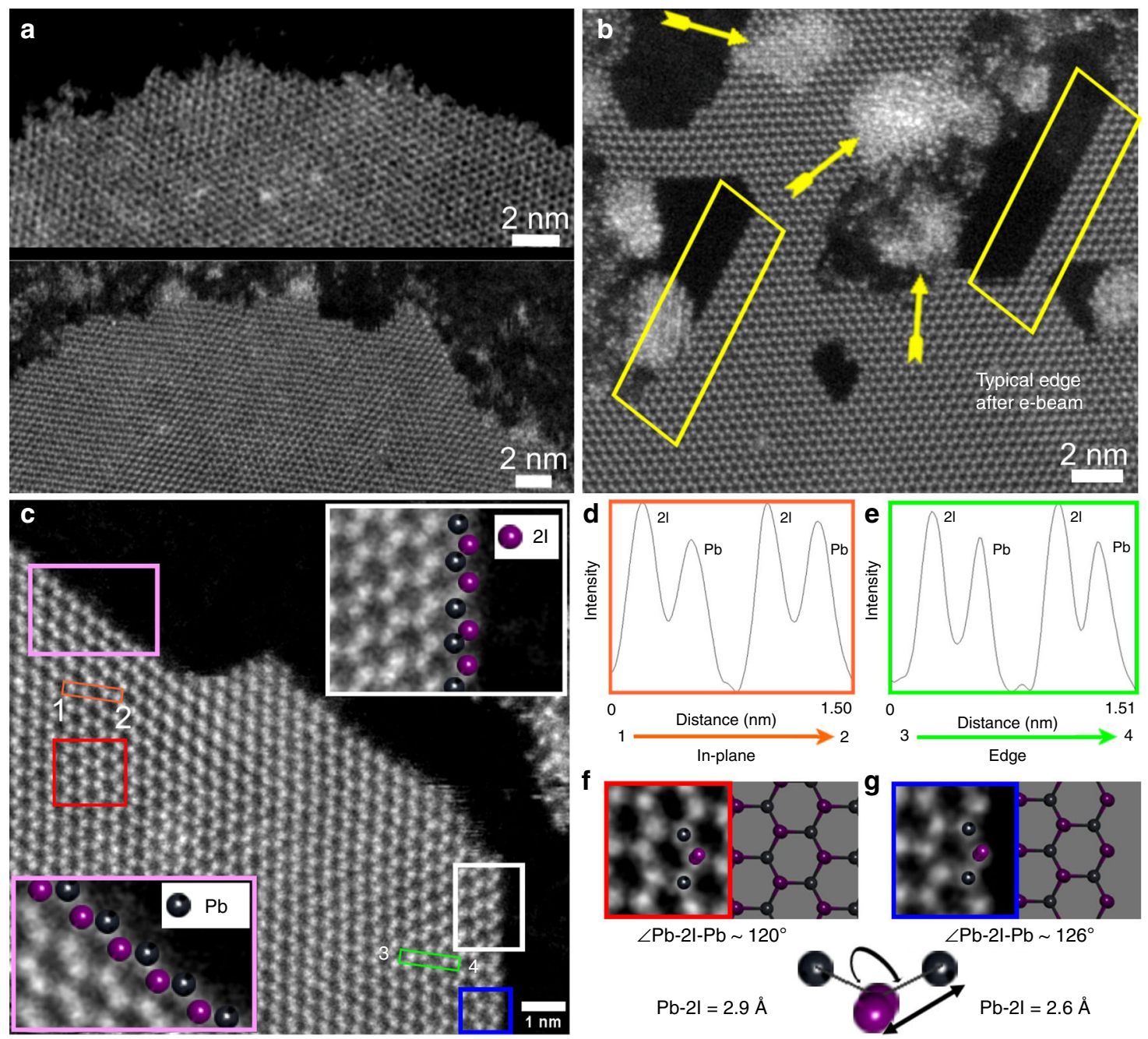

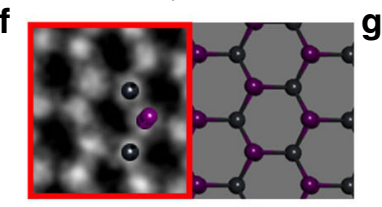

$\angle \mathrm{Pb}-2 \mathrm{I}-\mathrm{Pb} \sim 120^{\circ}$

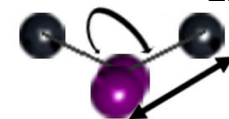

$\mathrm{Pb}-2 \mathrm{I}=2.9 \AA$

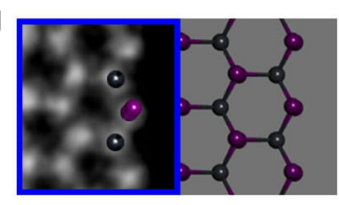

$\angle \mathrm{Pb}-2 \mathrm{I}-\mathrm{Pb} \sim 126^{\circ}$ $\mathrm{Pb}-2 \mathrm{I}=2.6 \AA$

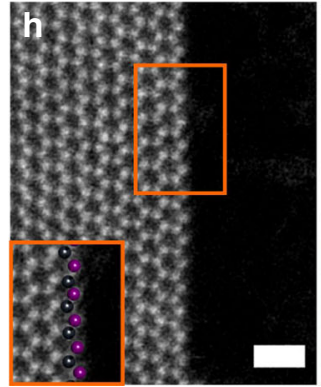

$t=0 \mathrm{~s}$

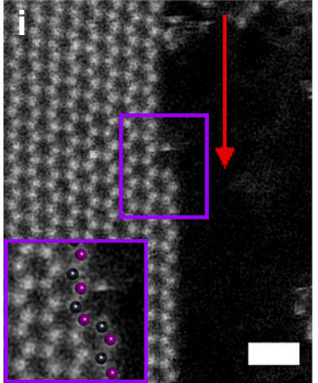

$t=30 \mathrm{~s}$

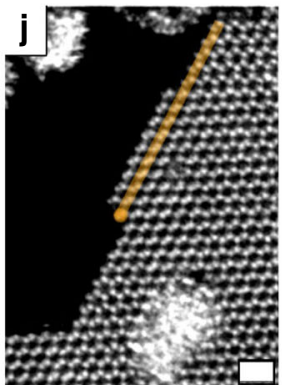

$t=0 \mathrm{~min}$

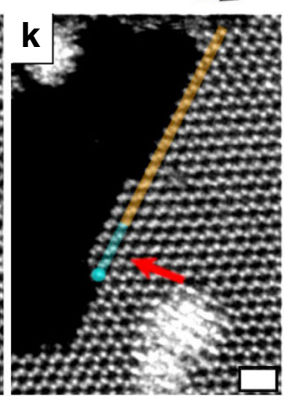

$t=1$ min

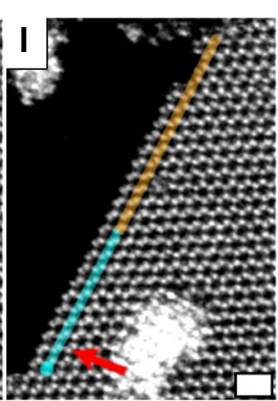

$t=2$ min

Fig. 4 Edge study of $\mathbf{P b}_{\mathbf{2}}$. ADF-STEM image of a typical intrinsic edges of two different monolayer $\mathrm{Pbl}_{2}$ flakes just after deposition on the graphene and b sharp zigzag-faceted edges of the monolayer $\mathrm{Pbl}_{2}$ flake after electron beam irradiation. The arrows show the clustering of the atoms after having been sputtered out of the edges. The yellow boxes show the faceted zigzag edge formation from the intrinsic edges after the damage from electron beam. c ADF-STEM images showing I- and Pb-terminated zigzag termination. The two insets in the image shown with white and pink boxes show the high resolution of the areas with same color annotation that have $\mathrm{I}$ - and Pb-terminated edges. The line profile of the regions marked in orange and green boxes confirms the position of the lead and iodide in the honeycomb structure. $\mathbf{d}$, e The line profile of the orange and green boxes shown in $\mathbf{c}$, in the direction of 1-2 and 3-4. $\mathbf{f}$ High-resolution ADF-STEM image of the area shown under red box in $\mathbf{c}$. $\mathbf{g}$ High-resolution ADF-STEM image of the area shown under blue box in $\mathbf{c}$. $\mathbf{h}$, $\mathbf{i}$ Time-lapse ADF-STEM image of the edge at $t=0 \mathrm{~s}$ and $t=30 \mathrm{~s}$ of electron beam exposure. The red arrow indicates the ejection of the electrons from the edges due to the damage from the electron beam, leading to 'unzipping' of the chain, maintaining the zigzag edge of the flakes. $\mathbf{j}-\mathbf{I}$ Timelapse series of ADF-STEM images recorded after $1 \mathrm{~min}$ of electron beam exposure. The yellow annotations show the edge-terminated bonds at the edge at $t=0 \mathrm{~s}$, which starts reconstructing in $\mathbf{k}$, annotated with blue. After $2 \mathrm{~min}$, (I) the edge was fully reconstructed, as shown with growing blue annotated region. The scale bars in $\mathbf{h}-\mathbf{I}$ correspond to $1 \mathrm{~nm}$. 


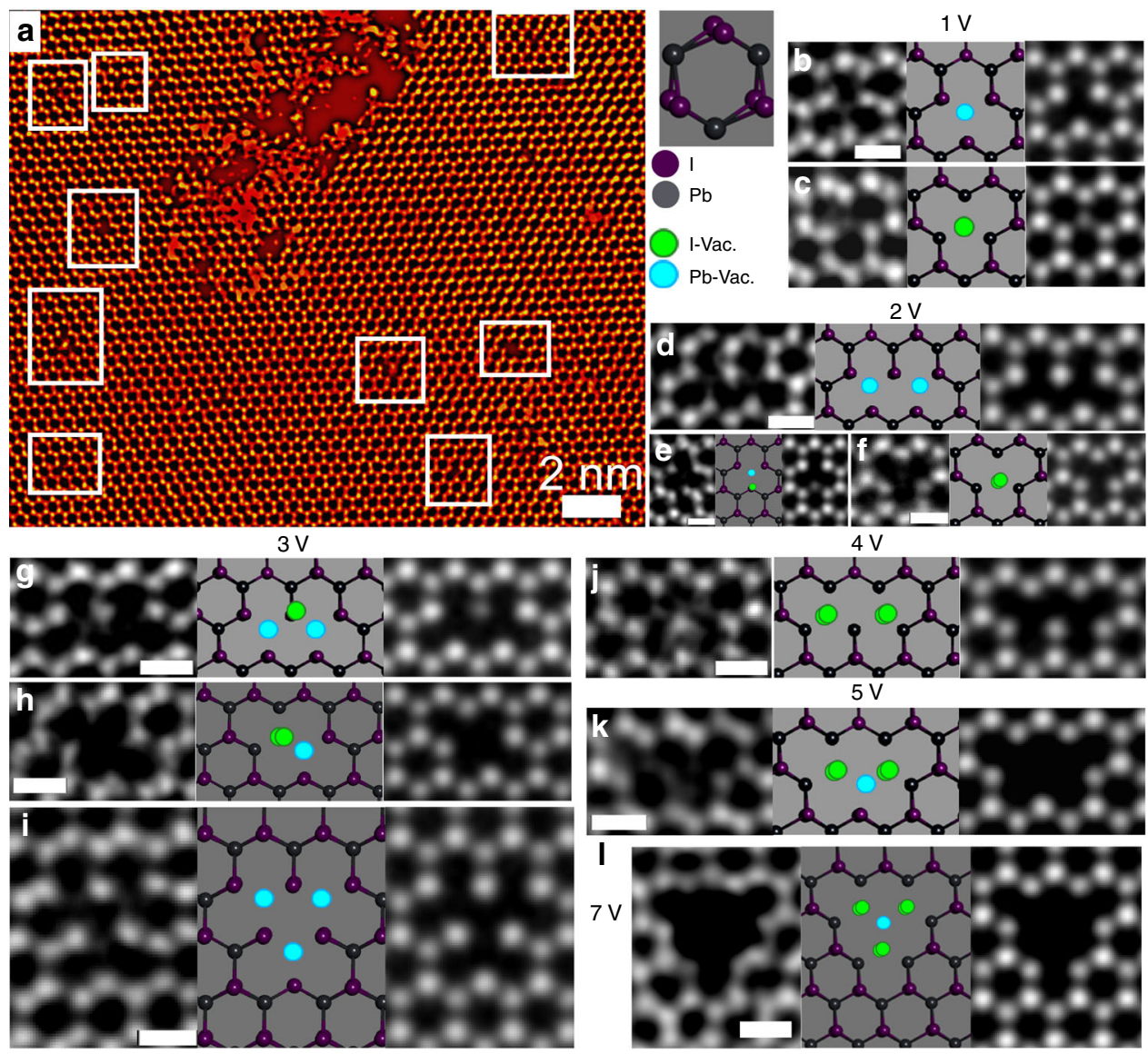

Fig. 5 Vacancy study in monolayer $\mathbf{P b l}_{2}$. a Low-magnification ADF-STEM image of monolayer $\mathrm{Pbl}_{2}$ region after electron beam exposure. The purple and gray spheres correspond to lead and iodide in the structure. The green and blue spheres correspond to the iodide and lead vacancies in the system. ADFSTEM images and their corresponding $1 \mathrm{H} \mathrm{Pbl}_{2}$ structured atomic model, as well as multislice simulations have been shown for $\mathbf{b}$ point defect with a missing lead atom, c point defect with a missing iodide atom, $\mathbf{d}$ double-point defect with two iodide atoms missing, e double-point defect where two lead atoms from adjacent crystal cells are lost, and $\mathbf{f}$ double-point defect with one lead and one iodide atoms missing. It can be observed that the single iodide has lower intensity than that of lead or two iodides, $\mathbf{g}$ three vacancies formed out of two lead and one iodide loss. It can be observed that the remaining single iodide in the middle has lower intensity than that of lead or two iodides, $\mathbf{h}$ three vacancy formed by the absence of three lead atoms from adjacent crystal cells, $\mathbf{i}$ another three vacancy formed by two iodide and one lead loss, $\mathbf{j}$ four vacancy defect formed by loss of two iodine from adjacent honeycomb structures, $\mathbf{k}$ five vacancies formed because of loss of four iodide atoms and a lead atom in a row, I bigger triangular defect formed because of the loss of one lead and six iodide atoms from the center. The scale bar in all the images indicate $0.5 \mathrm{~nm}$.

therefore prone to ionization effects from the electron beam. Several different types of multiple atom point vacancies are identified, ranging from two atoms (Fig, 5d, e) to the seven atom defect clusters (Fig. 5k). Vacancies did not appear to assembly into linear structures, as in TMDs, but instead aggregated to form clusters (Supplementary Note 16).

We also observed multiple occasions where the defect (Fig. 6) had one $\mathrm{Pb}$ atom in the center and five iodide atoms surrounding it, which was stable even under prolonged electron beam irradiation (Fig. 6a-d). Figure 6f, g,i, $j$ shows the proposed atomic model and the simulation of the two different configurations of this defect, (Fig. 6e, h) respectively. Since a single iodide atom is much lighter $(Z=53)$ than the single $\mathrm{Pb}$ atom $(Z=82)$, it has lower contrast in ADF-STEM. The line profiles in Fig. 6 confirm the presence of iodide atoms close to the lead atom in the center, causing the iodide peaks to be only partially resolved and forming a tail either side of the lead atom. Figure 6x shows the structure of the system, where $\mathrm{Pb}$ is coordinated with five other iodide atoms with inter-atomic distance varying from 1.4-2.3 $\AA$. We observed the formation of a stable four-membered ring around the lead atoms (Fig. 6f, i), where the lighter iodide atoms substitute the heavier lead atoms (Fig. 6f). Similar stable fourmembered ring configuration have also been reported for monolayer $\mathrm{MoS}_{2}$ structures ${ }^{59}$.

During electron beam irradiation, the vacancies migrated through the lattice and reconstructed. We performed DFT calculations for vacancy migration and found that the energy barrier for migration of lead atom, the in-plane migration of the iodide atom and the out-of-plane migration of iodide atom that corresponded to $0.32,0.22$, and $0.07 \mathrm{eV}$, respectively (Supplementary Note 17). The energy barrier for migration of the atoms on the surface is considerably lower than the energy imparted from the electron beam and thus explains the quick migration of the vacancies and defects in the system. Figure 7 is an example of a double iodide vacancy (Fig. 7b) and its migration (Fig. 7c) before subsequently healing to render the original structure in Fig. 7a, d. Defect healing occurred in point defects and also with in multiple atom vacancies, such as Fig. 7e-g, where a large defect with seven atoms missing (one $\mathrm{Pb}$ and six I) is healed.

Further electron beam exposure caused the vacancies grow larger and form nanopores in monolayer $\mathrm{PbI}_{2}$ crystals ${ }^{60,61}$. The nanopores undergo geometric reconstructions by atomic loss 


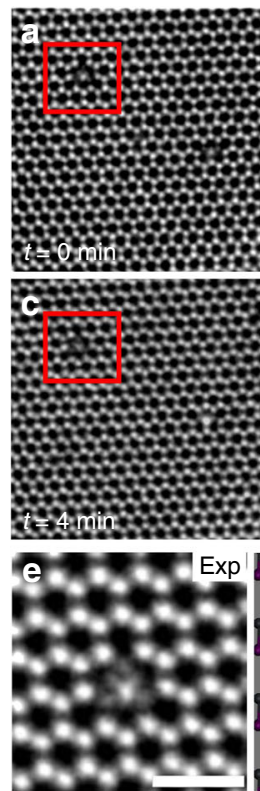

Exp

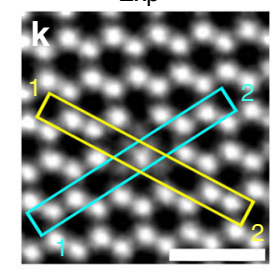

m

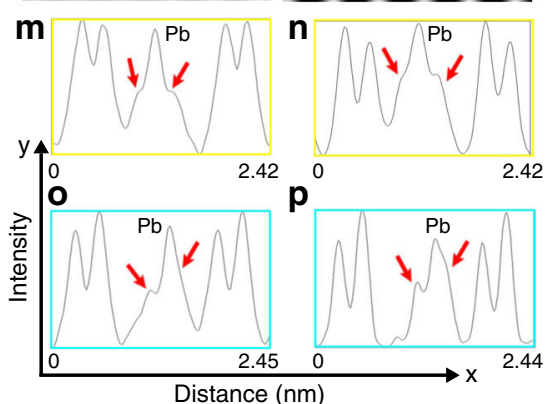

Distance (nm)

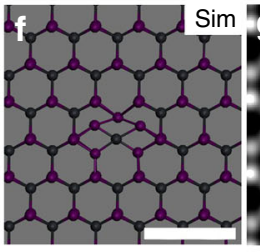

Sim

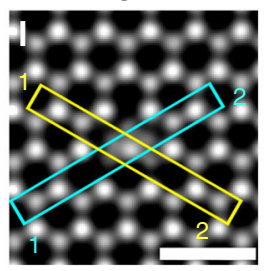

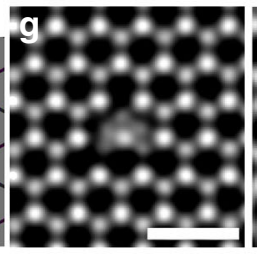

Exp

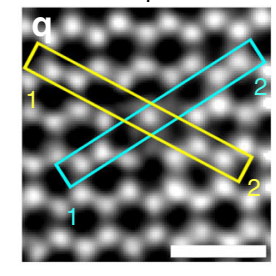

$\mathbf{S}$

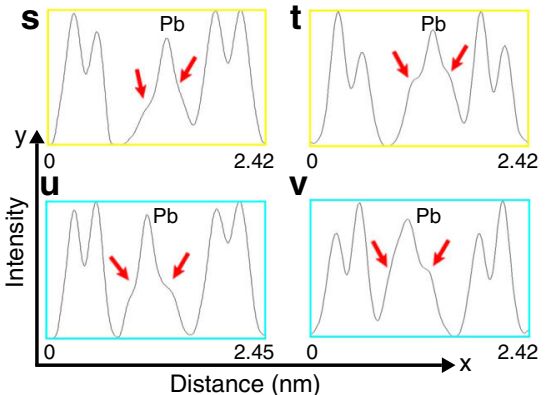

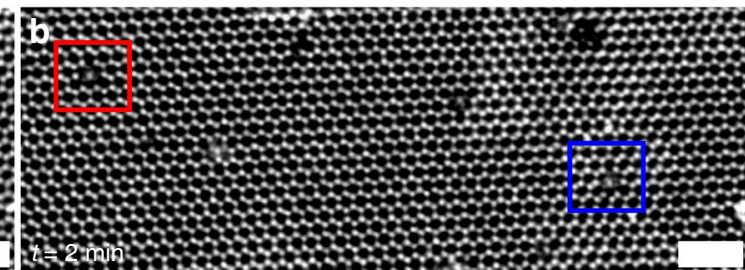

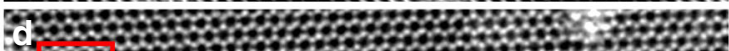

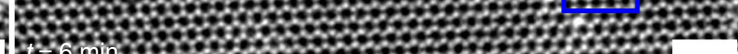

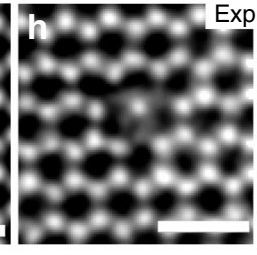

Sim
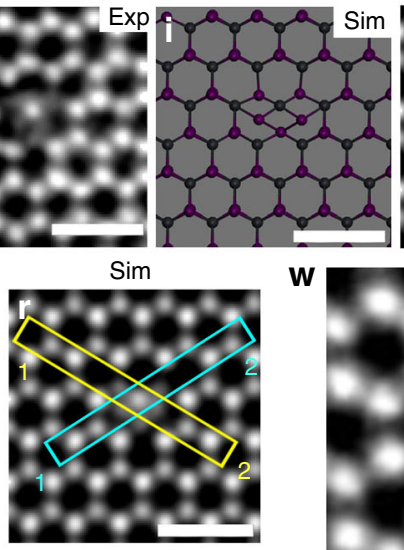

$\mathbf{W}$
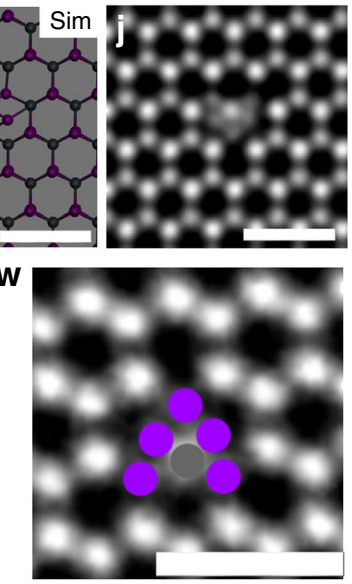

$\mathbf{X}$

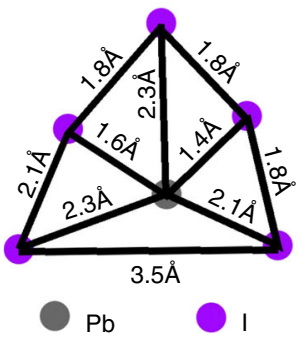

Fig. 6 Stable defect dynamics in $\mathbf{P b l}_{\mathbf{2}}$. a-d Time-lapse ADF-STEM images taken after every 2 min. The red and blue boxes show the two different

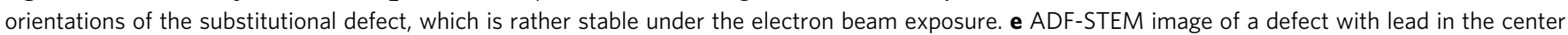
and surrounded by five other iodide atoms. $\mathbf{f}$ Atomic model that shows the atomic model of the corresponding experimental result. $\mathbf{g}$ Multislice image simulations based on the atomic model in $\mathbf{f}$, schematically illustrating the lower intensity of the single iodide atoms surrounding the lead, which agrees with

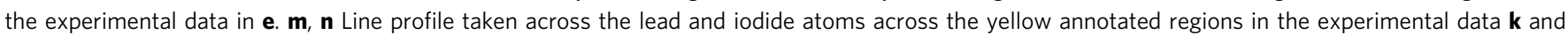
multislice simulated image in $\mathbf{I}$. The red arrow shows unresolved iodide peaks around the lead. o, $\mathbf{p}$ Line profile taken across the lead and iodide atoms across the blue annotated region in $\mathbf{k}$ and $\mathbf{I}$, respectively. The red arrow shows unresolved iodide peaks around the lead. $\mathbf{j}$, $\mathbf{h}$ ADF-STEM image of the opposite orientated substitutional defect. $\mathbf{i}$ Atomic model that shows the atomic model of the corresponding experimental result. $\mathbf{j}$ Multislice image

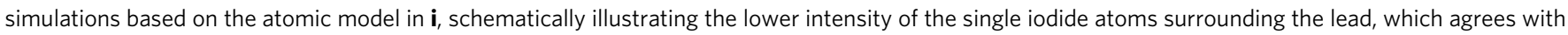
the experimental data in $\mathbf{h}$. s, $\mathbf{t}$ Line profile taken across the lead and iodide atoms across the yellow annotated regions in the experimental data $\mathbf{q}$ and multislice simulated image in $\mathbf{r}$. The red arrow shows unresolved iodide peaks around the lead. $\mathbf{u}, \mathbf{v}$ Line profile taken across the lead and iodide atoms across the blue annotated region in $\mathbf{q}$ and $\mathbf{r}$, respectively. The red arrow shows unresolved iodide peaks around the lead. $\mathbf{w}$ Annotated image of $\mathbf{e}$. $\mathbf{x}$ Distance between the different atoms shown in the annotated region in $\mathbf{w}$. Scale bars in a-d correspond to 2 nm, whereas in the rest of the images correspond to $1 \mathrm{~nm}$.

(Fig. 8a-p), but also sometimes gain atoms (Fig. 8o-p). Diffusing $\mathrm{Pb}$ and I atoms add to the edge sites of the nanopores to enable reconstruction. The nanopores in general adopt zigzag edge terminations and have triangular or hexagonal shape. In several cases, atoms migrate from one side of the nanopore to another side to change the nanopore shape. Figure $8 \mathrm{q}-\mathrm{u}$ shows a case, where a large triangular nanopore is formed by electron beam irradiation, and is then backfilled by its nearby atoms. Between Fig. $8 \mathrm{~s}$, $\mathrm{t}$, the top left section of the triangular nanopore has contracted by atoms filling it in from the nearby edge region. The red arrows indicate the possibility of migration of the atoms from one position to the other. The electron beam damages the material and forms the nanopore but at the same time, we are able to see the fast migration of atoms and the self-healing 


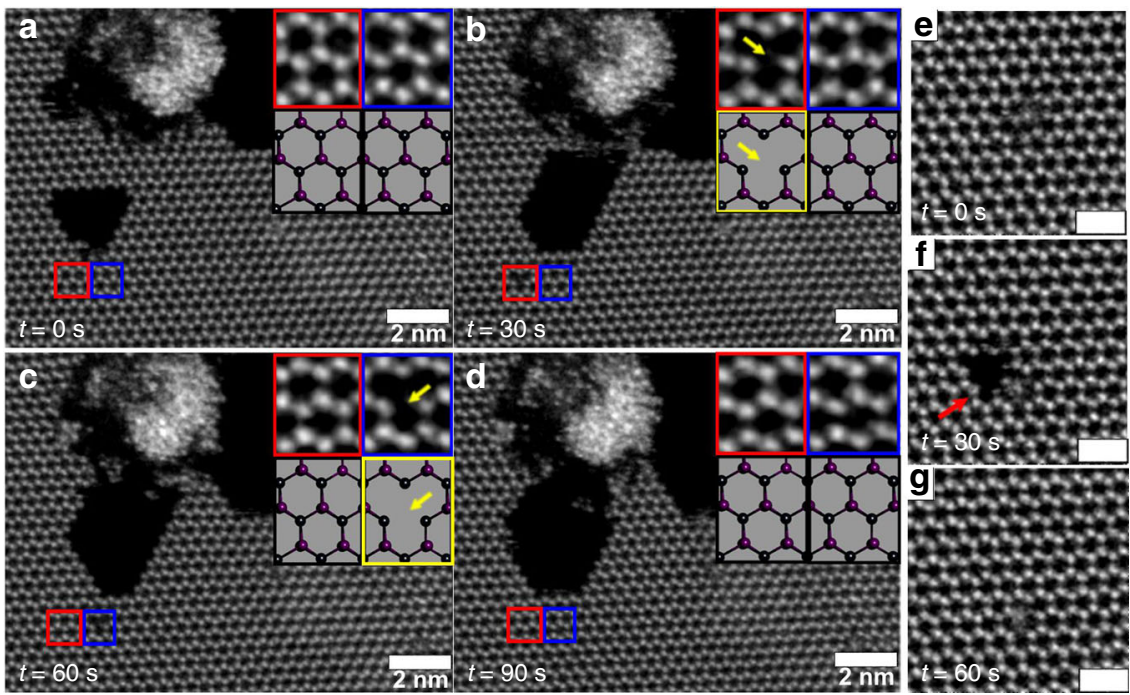

Fig. 7 Point defect formation, migration, and then reconstruction. a-d Time series of ADF-STEM images recorded after every $30 \mathrm{~s}$, of point defect formation, migration, and then reconstruction. The inset under red and blue boxes in the images are high-magnification images of the defects that have been marked with smaller red and blue boxes, respectively, in the same image. The inset figures in black and yellow boxes below the high-magnification insets are the corresponding atomic models. The yellow boxes and arrows in $\mathbf{b}$ and $\mathbf{c}$ highlights the formation of a point vacancy and its migration in the inset above, before finally reconstructing in $\mathbf{~} \mathbf{~}$. $\mathbf{e}-\mathbf{g}$ Time series of ADF-STEM images recorded at an accelerating voltage of $80 \mathrm{kV}$ after every $30 \mathrm{~s}$ of electron beam exposure. The red arrow in $\mathbf{f}$ shows the formation of seven vacancy defect, but it gets quickly reconstructed in image $\mathbf{g}$ after another $30 \mathrm{~s}$ of exposure. The scale bars in $\mathbf{e}-\mathbf{g}$ correspond to $1 \mathrm{~nm}$.

process in the material in real time. However, prolonged exposure to the electron beam ultimately leads excessive sputtering of atoms than the self-healing itself leading to a bigger nanopore formation. Similarly, in Fig. $8 \mathrm{q}-\mathrm{u}$, the self-healing is not perfect, but the nanopore is no longer present and vacancies have been eliminated by the migration to the edge.

We also observed the migration of entire nanopores as a single entity, up to $4 \mathrm{~nm}$ on the same monolayer flake. Figure $9 \mathrm{a}-\mathrm{i}$ is set of time lapsed images, taken every $30 \mathrm{~s}$, of hole formation and reconstruction as well as migration on the same monolayer flake upon beam exposure. The yellow boxes in Fig. 9g-i show the accumulation of the atoms at the edge and surface region next to the nanopore. The quick variation in the position and size of these holes clearly show how mobile the lead and iodide atoms are on the surface and continuously try to form new structures.

\section{Discussion}

We have successfully shown the liquid exfoliation of $\mathrm{PbI}_{2}$ using chloroform and formation of $>99 \%$ monolayer nanodisks. This approach has the potential to be scaled up to produce a large amount of exfoliated $\mathrm{PbI}_{2}$. Direct imaging of the monolayer $\mathrm{PbI}_{2}$ nanodisks is challenging using conventional TEM grids, and the use of graphene was critical for high-resolution ADF-STEM imaging. However, the van der Waals interactions with the underlying graphene lattice causes epitaxial alignment of the $\mathrm{PbI}_{2}$ and leads to the observation of the $1 \mathrm{H}$ crystal phase for $\mathrm{PbI}_{2}$, with no observation of $1 \mathrm{~T}$ phases on graphene. The relative lattice spacing of $\mathrm{PbI}_{2}$ and graphene results in preferential alignment of the $\mathrm{PbI}_{2}$ zigzag direction to the armchair direction of graphene to maximize the commensuration of the two lattices. Integrating monolayer $\mathrm{PbI}_{2}$ with other $2 \mathrm{D}$ materials provides a huge scope for many applications and the ability to manipulate its atomic monolayer structure provides a unique opportunity to tune its properties. Studying the influence of the substrate on the structure of $\mathrm{PbI}_{2}$ would hopefully make it easier to understand the complicated polytypes and stacking arrangement in $\mathrm{PbI}_{2}$.
ADF-STEM imaging of the $\mathrm{PbI}_{2}$ monolayers revealed highly mobile point vacancies and their growth into nanopores, which exhibited reconstructions and self-healing. Studying these vacancies in the $2 \mathrm{D}$ material is important for the foundation knowledge of the semiconducting properties for future work.

\section{Methods}

Chemical vapor deposition growth of graphene. Graphene was synthesized by CVD on a copper substrate. A piece of copper foil was first sonicated in a diluted hydrochloric acid solution $(1 \mathrm{~mol} / \mathrm{L})$ to remove the oxides on the copper surface. The copper foil was then sonicated in deionized water, acetone, and isopropanol for $5 \mathrm{~min}$ each to remove organic residues. To ensure uniformity of temperature during the whole growth process, the copper foil was positioned in the center of a furnace. A total of $1 \%$ methane in argon $\left(\mathrm{CH}_{4}\right), 25 \%$ hydrogen in argon $\left(\mathrm{H}_{2}\right)$, and $100 \%$ argon (Ar) were used for graphene synthesis. Before the reaction started, the system was first purged with $1000 \mathrm{sccm} \mathrm{Ar}, 500 \mathrm{sccm} \mathrm{H}$, and $100 \mathrm{sccm} \mathrm{CH}_{4}$ for $30 \mathrm{~min}$. The furnace was then heated to $1060^{\circ} \mathrm{C}$ with a ramp rate of $50{ }^{\circ} \mathrm{C} / \mathrm{min}$ accompanied by a flow of $500 \mathrm{sccm} \mathrm{Ar}$ and $100 \mathrm{sccm} \mathrm{H} \mathrm{H}_{2}$. When the temperature reached $1060^{\circ} \mathrm{C}$, the copper was then annealed with the same flow rate for $1 \mathrm{~h}$. Following the annealing process, the synthesis was carried out at $1060^{\circ} \mathrm{C}$ for $1 \mathrm{hr}$ with a flow of $500 \mathrm{sccm} \mathrm{Ar,} 100 \mathrm{sccm} \mathrm{H}_{2}$, and $5 \mathrm{sccm} \mathrm{CH}_{4}$. The furnace was removed from the sample, which allowed the sample to be fast cooled to room temperature.

Liquid-phase exfoliation. $\mathrm{PbI}_{2}$ powder from Sigma-Aldrich (99\% purity) was added to chloroform to form a solution of concentration $1 \mathrm{mg} / \mathrm{ml}(\sim 0.2 \mathrm{mM})$. The solution was sonicated in an unheated ultrasonic water bath (Ultrasonics effective output of $200 \mathrm{~W}$; Ultrasonics peak power of $600 \mathrm{~W}$; Ultrasonics operating frequency $30-40 \mathrm{kHz}$ ) for $\sim 30 \mathrm{~min}$ to make sure that all the lead iodide had exfoliated and dispersed in the solution. The dispersion was then left to stand for $24 \mathrm{~h}$ to let the bulk particles to settle down at the bottom of the container. Then, the supernatant of the dispersion (from the top) was extracted with a pipette and used for preparing TEM samples.

TEM sample preparation. Graphene was transferred onto a lacey carbon copper 400 mesh TEM grid using a poly (methyl methacrylate (PMMA) support and then the PMMA removed by acetone. It was then subsequently cleaned by $\mathrm{Ar} / \mathrm{H}_{2}$ and vacuum annealing. A few drops (10-20 $\mu$ leach) were taken from the supernatant of the dispersed solution of $1 \mathrm{mg} / \mathrm{ml}$ concentration of $\mathrm{PbI}_{2}$ in $\mathrm{CHCl}_{3}$ and dropcasted on to the TEM grid containing suspended graphene. The TEM holder was then baked in high vacuum at $60^{\circ} \mathrm{C}$ to remove all the solvents and other impurities from the surface. 

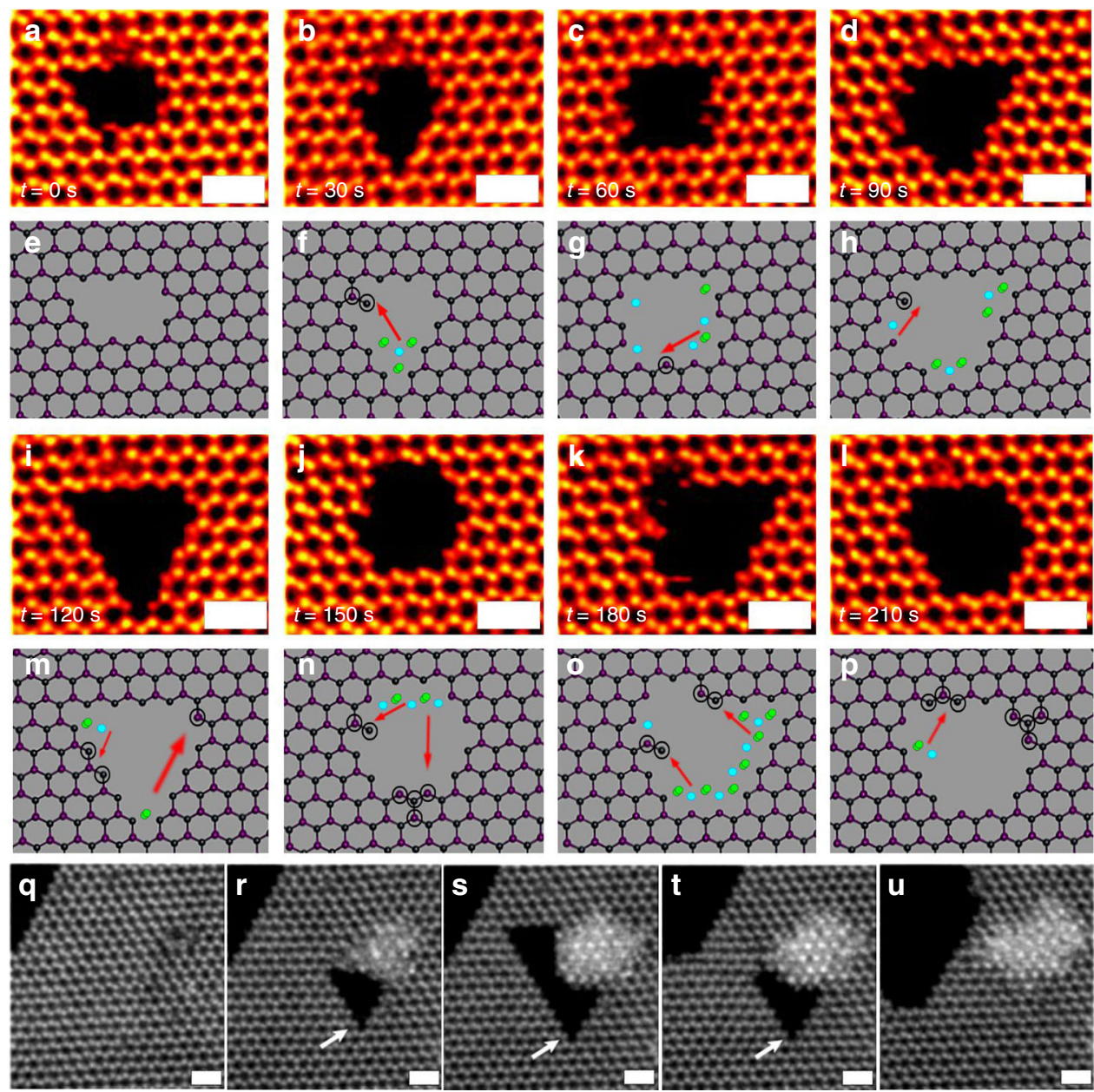

$$
t=0 \min
$$

$$
t=1 \mathrm{~min}
$$

$t=2 \min$

$t=3 \mathrm{~min}$

$t=4 \min$

Fig. 8 Nanopore geometric reconstructions. a-p Time series of ADF-STEM images, and their corresponding atomic models. Holes can be seen to change its shape in monolayer $\mathrm{Pbl}_{2}$ flake. The blue atoms correspond the lead defect region and the green corresponds to the iodide defect region. The black circles around the atoms in the atomic models correspond to the new atomic bond in the structure as compared from the previous frame. q-u Time series of ADFSTEM images recorded after every $1 \mathrm{~min}$ of electron beam exposure. The defect starts forming after $1 \mathrm{~min}$, in image $\mathbf{r}$ and more atoms are ejected to form a bigger defect in $\mathbf{s}$. Then the atoms at the edge starts ejecting out in $\mathbf{t}$ and reconstruction of the flake starts occurring leading to again defect-free surface in u. The scale bar in all images corresponds to $1 \mathrm{~nm}$.

Scanning transmission electron microscopy. Room temperature ADF-STEM imaging was performed using a JEOL ARM200F located at the David Cockayne Centre for Electron Microscopy (DCCEM) within the Department of Materials at the University of Oxford. An acceleration voltage of $80 \mathrm{kV}$ was used for taking all the images, unless stated otherwise. Imaging conditions used a $30 \mu \mathrm{m}$ condenser lens (CL) aperture with a convergence semiangle of $22.5 \mathrm{mrad}$ and a beam current of $35 \mathrm{pA}$. The acquisition angles for these images was $70-220 \mathrm{mrad}$.

Data processing. Images were processed using the ImageJ software. Atomic models were constructed using Accelrys Discovery Studio Visualizer. Multislice image simulations were performed using JEMS. $\mathrm{PbI}_{2}$ crystal structure was drawn using Crystal Maker Version 10.3.1.

DFT calculation. The structural, energetic, and defect migration calculations in this work are based on DFT implemented in the Vienna Ab-initio Simulation Package $(\text { VASP })^{62,63}$. A $10 \times 10 \times 1$ supercell is used for calculating the sputtering energy and defect migration barriers, and two more different systems are designed to probe the short-range angle dependence of stacking energy: a hexagonal $\mathrm{PbI}_{2}$ flake (side length four $\mathrm{PbI}_{2}$ unit cells, $1.8 \mathrm{~nm}$ ) on continuous graphene substrate. The systems are varied with different angles between $\mathrm{PbI}_{2}$ and graphene (i.e., armchair to zigzag stacking order). The elements are represented by projector-augmented wave potentials with $500 \mathrm{eV}$ energy cutoff and the four $\left(6 s^{2} 6 p^{2}\right)$ valence electrons for $\mathrm{Pb}$, seven $\left(5 s^{2} 5 p^{5}\right)$ for $\mathrm{I}$, and four $\left(2 s^{2} 2 p^{2}\right)$ for $\mathrm{C}$ are treated explicitly $\mathrm{y}^{64,65}$. Initial relaxation and energetics are calculated via the generalized gradient approximation, Perdew-Burke-Ernzerhof, augmented by DFT-D3 method with Becke-Jonson damping for the interlayer dispersive interaction ${ }^{66,67}$. The first Brillouin zone is sampled by the tetrahedron method on a $4 \times 4 \times 1 k$-mesh for the $10 \times 10 \times$ 1 supercell, and only gamma point for the angle-dependence studies. All structures are relaxed until the force on each atom is $<0.01 \mathrm{meV} / \AA$. The sputtering energy is defined by the energy difference between the crystalline structure and the one with a vacancy, for instance, the I sputtering energy is $\Delta E_{\mathrm{I}}=E_{\text {crystal }}-\left(E_{V_{\mathrm{I}}}+E_{\mathrm{I}}\right)$. The energy tolerance is set to 0.0001 for all energy calculations. The minimum energy paths for defect migration are determined by the nudged elastic band (NEB) method ${ }^{68,69}$. A total of $10 \times 10$ supercells and $4 \times 4 k$-point grids are employed, and a criterion of $0.03 \mathrm{eV} / \AA$ is set for the force convergence. For $V_{\mathrm{I}}$ migration, two pathways are considered: in-plane and out-of-plane directions, and only in-plane $V_{\mathrm{Pb}}$ migration is considered. The starting and end points for NEB search are prepared by removing the corresponding atoms from the relaxed pristine structure, and then further relaxed. The six intermediate images are interpolated by the Vasp TST toolkit ${ }^{70}$. The converged energy paths are the fitted by cubic spline functions.

Supporting information. Effect of sonication duration and dropcasting, monolayer and bilayer $\mathrm{PbI}_{2}$ intensity contrast, graphene sample without $\mathrm{PbI}_{2}$, different sizes of monolayer $\mathrm{PbI}_{2}$, proof of monolayer flakes instead of bilayer $\mathrm{AA}^{\prime} 1 \mathrm{H}$ structure, $\mathrm{T}$ phase structure on lacey carbon TEM grid, ADF-STEM images of structural transformation immediately after dropcasting on graphene, Moire pattern of $1 \mathrm{~T}$ and $1 \mathrm{H}$ atomic models on graphene, zigzag alignment of $\mathrm{PbI}_{2}$ with graphene after edge etching, relative alignment of time frame of $\mathrm{PbI}_{2}$ etching from Fig. 4, line profile from Fig. 5, DFT calculation of sputtering energy of lead and iodide, accumulation of lead particles after etching, and DFT calculation of vacancy migration. 

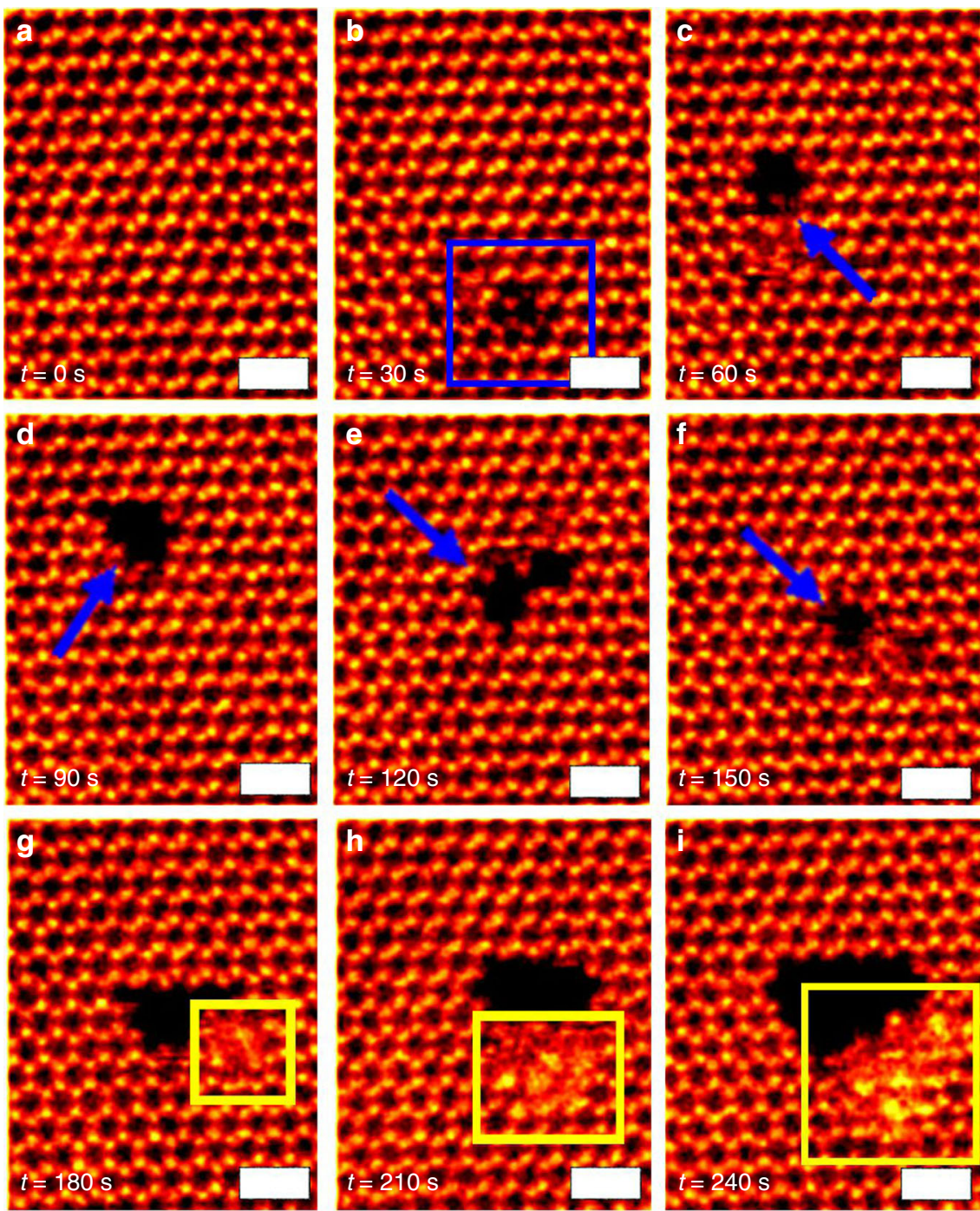

Fig. 9 Nanopore migration. a-i Time series of ADF-STEM images recorded of nanopores changing form, as well as migrating large distances within singlemonolayer $\mathrm{Pbl}_{2}$ flake. The scale bar corresponds to $1 \mathrm{~nm}$.

\section{Data availability}

Experimental procedures and characterization data are available within this article and its Supplementary Information file. The data that support the findings of this study are available from the corresponding authors upon reasonable request.

Received: 23 May 2019; Accepted: 6 January 2020;

Published online: 10 February 2020

\section{References}

1. Wang, Q. H., Kalantar-zadeh, K., Kis, A., Coleman, J. N. \& Strano, M. S. Transition metal dichalcogenides. Nat. Nanotech. 7, 699-712 (2012).

2. Mak, K. F., He, K., Shan, J. \& Heinz, T. F. Control of valley polarization in monolayer MoS2 by optical helicity. Nat. Nanotech. 7, 494-498 (2012).

3. Zeng, H., Dai, J., Yao, W., Xiao, D. \& Cui, X. Valley polarization in MoS2 monolayers by optical pumping. Nat. Nanotech. 7, 490-493 (2012).

4. Elias, D. C. et al. Dirac cones reshaped by interaction effects in suspended graphene. Nat. Phys. 7, 701-704 (2011).

5. Radisavljevic, B., Radenovic, A., Brivio, J., Giacometti, V. \& Kis, A. Single-layer MoS2 transistors. Nat. Nanotech. 6, 147-150 (2011).

6. Amani, M. et al. Vapor deposition electrical performance of monolayer MoS2 field-effect transistors prepared by chemical vapor deposition. Appl. Phys. Lett. 102, 193107 (2013).
7. Ganatra, R. \& Zhang, Q. Few-layer MoS2: a promising layered semiconductor ACS Nano 5, 4074-4099 (2014).

8. Lv, R. et al. Transition metal dichalcogenides and beyond: synthesis, properties, and applications of single- and few-layer nanosheets. Acc. Chem. Res. 48, 56-64 (2015)

9. Schwierz, F., Pezoldt, J. \& Granzner, R. Two-dimensional materials and their prospects in transistor electronics. Nanoscale 7, 8261-8283 (2015).

10. Zhou, M., Duan, W., Chen, Y. \& Du, A. Single layer lead iodide: computational exploration of structural, electronic and optical properties, strain induced band modulation and the role of spin-orbital-coupling. Nanoscale 7, 15168-15174 (2015).

11. Zhong, $\mathrm{M}$. et al. Large-scale $2 \mathrm{D} \mathrm{PbI} 2$ monolayers: experimental realization and their indirect band-gap related properties. Nanoscale 9, 3736-3741 (2017).

12. Toulouse, A. S. et al. Frenkel-like Wannier-Mott Excitons in few-layer PbI2. Phys. Rev. B 19, 165308 (2015).

13. Arbiol, J. et al. Whispering gallery mode lasing from hexagonal shaped layered lead iodide. ACS Nano 9, 687-695 (2015).

14. Zhengqi, S. \& Jayatissa, A. H. Perovskites-based solar cells: a review of recent progress, materials and processing methods. Materials (Basel) 11, 729 (2018).

15. Niu, G., Guo, X. \& Wang, L. Review of recent progress in chemical stability of perovskite solar cells. J. Mater. Chem. A 3, 8970-8980 (2015).

16. Heo, J. Development of a stable and sensitive semiconductor detector by using a mixture of lead(II) iodide and lead monoxide for NDT radiation dose detection. JINST 13, C03023 (2018).

17. Zhu, X. H., Wei, Z. R., Jin, Y. R. \& Xiang, A. P. Growth and characterization of a PbI2 Single crystal used for gamma ray detectors. Cryst. Res. Technol. 42, 456-459 (2007). 
18. Greenspan, J.; Laperriere, L.; Bubon, O.; Reznik, A. Amorphous and polycrystalline photoconductors for direct conversion flat panel X-ray image sensors. Sensors (Basel) 11, 5112-5157 (2011).

19. Beckmann, P. A. A review of polytypism in lead iodide. Cryst. Res. Technol. 45, 455-460 (2010).

20. Han, M. et al. Two-step vapor deposition of self-catalyzed large-size PbI2 nanobelts for high-performance photodetectors. J. Mater. Chem. C 21, 5746-5753 (2018).

21. Lan, C. et al. Large-scale synthesis of freestanding layer-structured $\mathrm{PbI} 2$ and MAPbI3 nanosheets for high-performance photodetection. Adv. Mat. 29, 1702759 (2017).

22. Tan, M. et al. 2D lead dihalides for high-performance ultraviolet photodetectors and their detection mechanism investigation. Small 13, 1-8 (2017).

23. Zheng, W. et al. High-crystalline $2 \mathrm{D}$ layered PbI2 with ultrasmooth surface: liquid-phase synthesis and application of high-speed photon detection. Adv. Electron. Mater. 2, 1-8 (2016).

24. Rybak, O. et al. Luminescent spectra of PbI single crystals doped by $3 \mathrm{~d}$-metal impurities. J. Lumin. 79, 257-267 (1998).

25. Chang, Y. \& James, R. B. Phonon dispersion and polar-optical scattering in 2H PbI2. Phys. Rev. B 55, 8219-8225 (1997).

26. Cabana, L. et al. Synthesis of PbI2 single-layered inorganic nanotubes encapsulated within carbon nanotubes. Adv. Mater. 26, 2016-2021 (2014).

27. Fan, Q. et al. Liquid exfoliation of two-dimensional PbI2 nanosheets for ultrafast photonics. ACS Photonics 6, 1051-1057 (2019).

28. Nicolosi, V.; Chhowalla, M.; Kanatzidis, M. G.; Strano, M. S. \& Coleman, J. N. Liquid exfoliation of layered materials. Science, 340, 1226419 (2013).

29. Tao, H. et al. Scalable exfoliation and dispersion of two-dimensional materials-an update. Phys. Chem. Chem. Phys. 19, 921-960 (2017).

30. Huo, C., Yan, Z., Song, X. \& Zeng, H. 2D materials via liquid exfoliation: a review on fabrication and applications. Sci. Bull. 60, 1994-2008 (2015).

31. May, P., Khan, U., Hughes, J. M. \& Coleman, J. N. Role of Solubility parameters in understanding the steric stabilization of exfoliated twodimensional nanosheets by adsorbed polymers. J. Phys. Chem. C 116, 11393-11400 (2012).

32. Shen, J. et al. Liquid phase exfoliation of two-dimensional materials by directly probing and matching surface tension components. Nano Lett. 15, 5449-5454 (2015).

33. Hernandez, Y., Lotya, M., Rickard, D., Bergin, S. D. \& Coleman, J. N. Measurement of multicomponent solubility parameters for graphene facilitates solvent discovery. Langmuir 3, 3208-3213 (2010).

34. Manna, K., Huang, H. N., Li, W. T., Ho, Y. H. \& Chiang, W. H. Toward understanding the efficient exfoliation of layered materials by water-assisted cosolvent liquid-phase exfoliation. Chem. Mater. 28, 7586-7593 (2016).

35. Wang, M. et al. Surface tension components ratio: an efficient parameter for direct liquid phase exfoliation. ACS Appl. Mater. Interfaces 9, 9168-9175 (2017).

36. $\mathrm{Li}, \mathrm{H}$. et al. Atomic structure and dynamics of single platinum atom interactions with monolayer MoS2. ACS Nano 11, 3392-3403 (2017)

37. Wang, S. et al. Epitaxial templating of two-dimensional metal chloride nanocrystals on monolayer molybdenum disulfide. ACS Nano 11, 6404-6415 (2017).

38. Zhou, S. et al. Atomic structure and dynamics of defects in 2D MoS2 bilayers. ACS Omega 2, 3315-3324 (2017)

39. Brogan, M. A., Blake, A. J., Wilson, C. \& Gregory, D. H. Magnesium diiodide, MgI2. Acta Cryst. C59, i136-i138 (2003).

40. Patnaik, P. Handbook of Inorganic Chemicals. (McGraw-Hill, 2003)

41. Clever, H. L. \& Johnston, F. J. The solubility of some sparingly soluble lead salts: an evaluation of the solubility in water and aqueous electrolyte solution. J. Phys. Chem. Ref. Data 9, 751 (1980).

42. Hamill, J. C. \& Loo, Y. Influence of solvent coordination on hybrid organic inorganic perovskite formation. ACS Energy Lett. 3, 92-97 (2018).

43. Zhou, K., Mao, N., Wang, H., Peng, Y. \& Zhang, H. A mixed-solvent strategy for efficient exfoliation of inorganic graphene analogues. Angew. Chem. Int. Ed. 50, 10839-10842 (2011).

44. Cunningham, G. et al. Solvent exfoliation of transition metal dichalcogenides: dispersibility of exfoliated nanosheets varies only weakly between compounds. ACS Nano 6, 3468-3480 (2012).

45. Cataldo, F. A revision of the gutmann donor numbers of a series of phosphoramides including tepa. Eur. Chem. Bull. 4, $92-97$ (2015).

46. Huggins, C. M., Pimentel, G. C. \& Shoolery, J. N. Proton magnetic resonance studies of chloroform in solution: evidence for hydrogen bonding. J. Chem. Phys. 23, 1244-1247 (1955).

47. McCleallan, A. L., Nicksic, S. W. \& Guffy, J. C. NMR study of hydrogen bonding between chloroform and dimethyl. J. Mol. Spectr. 348, 340-348 (1963).
48. Wang, S., Robertson, A., Warner, J. H. \& Robertson, A. Atomic structure of defects and dopants in 2D layered transition metal dichalcogenides. Chem. Soc. Rev. 47, 6764-6794 (2018).

49. Bacaksiz, C. \& Sahin, H. Single layer PbI2: hydrogenation-driven reconstructions. RSC Adv. 6, 89708-89714 (2016).

50. Zhao, W. et al. Metastable MoS2: crystal structure, electronic band structure, synthetic approach and intriguing physical properties. Chem. Eur. J . 24 15942-15954 (2018).

51. Ezawa, M. Peculiar width dependence of the electronic properties of carbon nanoribbons. Phys. Rev. B 2005, 1-8 (2006).

52. Kawai, T. Graphitic ribbons without hydrogen-termination: electronic structures and stabilities. Phys. Rev. B 62, 349-352. (2000).

53. Li, Y., Zhou, Z., Zhang, S. \& Chen, Z. MoS2 nanoribbon: high stability and unusual electronic and magnetic properties. J. Am. Chem. Soc. 130, 16739-16744 (2008).

54. Butler, S. Z. et al. Progress, challenges, and opportunities in two-dimensional materials beyond graphene. ACS Nano 7, 2898-2926 (2013).

55. Wang, S. et al. Atomically sharp crack tips in monolayer MoS2 and their enhanced toughness by vacancy defects. ACS Nano 10, 9831-9839 (2016).

56. Wang, S., Sawada, H., Allen, C. S., Kirkland, A. I. \& Warner, J. H. Orientation dependent interlayer stacking structure in bilayer MoS2 domains. Nanoscale $\mathbf{9}$, 13060-13068 (2017).

57. Sang, $\mathrm{X}$. et al. Unocic, in situ edge engineering in two-dimensional transition metal dichalcogenides. Nat. Commun. 9, 2051 (2018).

58. Egerton, R. F., Li, P. \& Malac, M. Radiation damage in the TEM and SEM. Micron 35, 399-409 (2004)

59. Zhou, S. et al. Atomically sharp interlayer stacking shifts at anti-phase grain boundaries in overlapping MoS2 secondary layers. Nanoscale 10, 16692-16702 (2018).

60. Chen, Q. et al. Ultralong 1D vacancy channels for rapid atomic migration during $2 \mathrm{D}$ void formation in monolayer MoS2. ACS Nano 12, 7721-7730 (2018).

61. Russo, C. J. \& Golovchenko, J. A. Atom-by-atom nucleation and growth of graphene nanopores. PNAS 109, 5953-5957 (2012).

62. Kresse, G. \& Furthmüller, J. Efficient iterative schemes for ab initio total-energy calculations using a plane-wave basis set. Phys. Rev. B 54, 11169-11186 (1996)

63. Kresse, G. \& Furthmüller, J. Efficiency of ab-initio total energy calculations for metals and semiconductors using a plane-wave basis set. Comput. Mater. Sci. 6, 15-50 (1996).

64. Blöchl, P. E. Projector augmented-wave method. Phys. Rev. B 50, 17953-17979 (1994)

65. Kresse, G. \& Joubert, J. From ultrasoft pseudopotentials to the projector augmented wave method. Phys. Rev. B 59, 1758 (1999).

66. Perdew, J. P., Burke, K. \& Ernzerhof, M. Generalized gradient approximation made simple. Phys. Rev. Lett. 77, 3865 (1996).

67. Grimme, S., Ehrlich, S. \& Goerigk, L. Effect of the damping function in dispersion corrected density functional theory. J. Comp. Chem. 32, 1456 (2011).

68. Sheppard, D. \& Henkelman, G. Paths to which the nudged elastic band converges. J. Comp. Chem. 32, 1769-1771 (2011).

69. Sheppard, D., Terrell, R. \& Henkelman, G. Optimization methods for finding minimum energy paths. J. Chem. Phys. 128, 134106 (2008).

70. Henkelman, G. \& Jónsson, H. Improved tangent estimate in the nudged elastic band method for finding minimum energy paths and saddle points. J. Chem. Phys. 113, 9978-9985 (2000).

\section{Acknowledgements}

J.H.W. thanks the support from an ERC Consolidator Grant (LATO 725258). S.S. thanks the joint committee of Linacre College and MPLS division of the University of Oxford for financial support through the Women in Science Scholarship and EPSRC studentship. The authors acknowledge use of characterization facilities within the David Cockayne Centre for Electron Microscopy, Department of Materials, University of Oxford.

\section{Author contributions}

S.S. and J.H.W conceived the idea for the project, designed the experiments and analysis procedure. S.S. carried out all the chemical synthesis and experiments. S.S. carried out analysis of data and wrote the manuscript with contributions from J.H.W. Y.S. synthesized monolayer graphene. T.Z. and A.F.L carried out the DFT calculation. J.C.G. and K.P. provided materials for the experiments and helped with scientific discussions. J.H.W. supervised the project. All authors discussed the results and commented on the manuscript.

\section{Competing interests}

The authors declare no competing interests. 


\section{Additional information}

Supplementary information is available for this paper at https://doi.org/10.1038/s41467020-14481-z.

Correspondence and requests for materials should be addressed to J.H.W.

Peer review information Nature Communications thanks the anonymous reviewer(s) for their contribution to the peer review of this work. Peer reviewer reports are available.

Reprints and permission information is available at http://www.nature.com/reprints

Publisher's note Springer Nature remains neutral with regard to jurisdictional claims in published maps and institutional affiliations. (c) Open Access This article is licensed under a Creative Commons Attribution 4.0 International License, which permits use, sharing, adaptation, distribution and reproduction in any medium or format, as long as you give appropriate credit to the original author(s) and the source, provide a link to the Creative Commons license, and indicate if changes were made. The images or other third party material in this article are included in the article's Creative Commons license, unless indicated otherwise in a credit line to the material. If material is not included in the article's Creative Commons license and your intended use is not permitted by statutory regulation or exceeds the permitted use, you will need to obtain permission directly from the copyright holder. To view a copy of this license, visit http://creativecommons.org/licenses/by/4.0/.

(C) The Author(s) 2020 